\title{
Reduced Order Models \\ for Distributed Adaptive Monitoring of Atmospheric Dispersion Processes
}

Tobias Ritter $^{1,2}$, Stefan Ulbrich ${ }^{1,3}$ and Oskar von Stryk ${ }^{1,2}$

$\bowtie$ ritter@gsc.tu-darmstadt.de

${ }^{1}$ Graduate School of Computational Engineering, ${ }^{2}$ Department of Computer Science, ${ }^{3}$ Department of Mathematics, TU Darmstadt

\section{Monitoring Atmospheric Dispersion}

Air pollution due to accidental releases of chemical and radioactive substances can have a critical impact on human health and well-being. Therefore, monitoring of the atmospheric dispersion of these substances is essential for disaster response. The goal is to obtain repeatedly updated estimates of the current process state and of characteristic process parameters.

For this purpose, predictions from a process model and measurements from a mobile sensor network are combined to obtain good quality estimates. Furthermore, the sensor vehicles are guided to positions where a higher amount of information can be obtained (Adaptive Observation Strategy (AO)).

\section{$\rightarrow$ Process Simulation Prediction

Sensor Vehicles Measurements

Control Inputs

Vehicle Controller

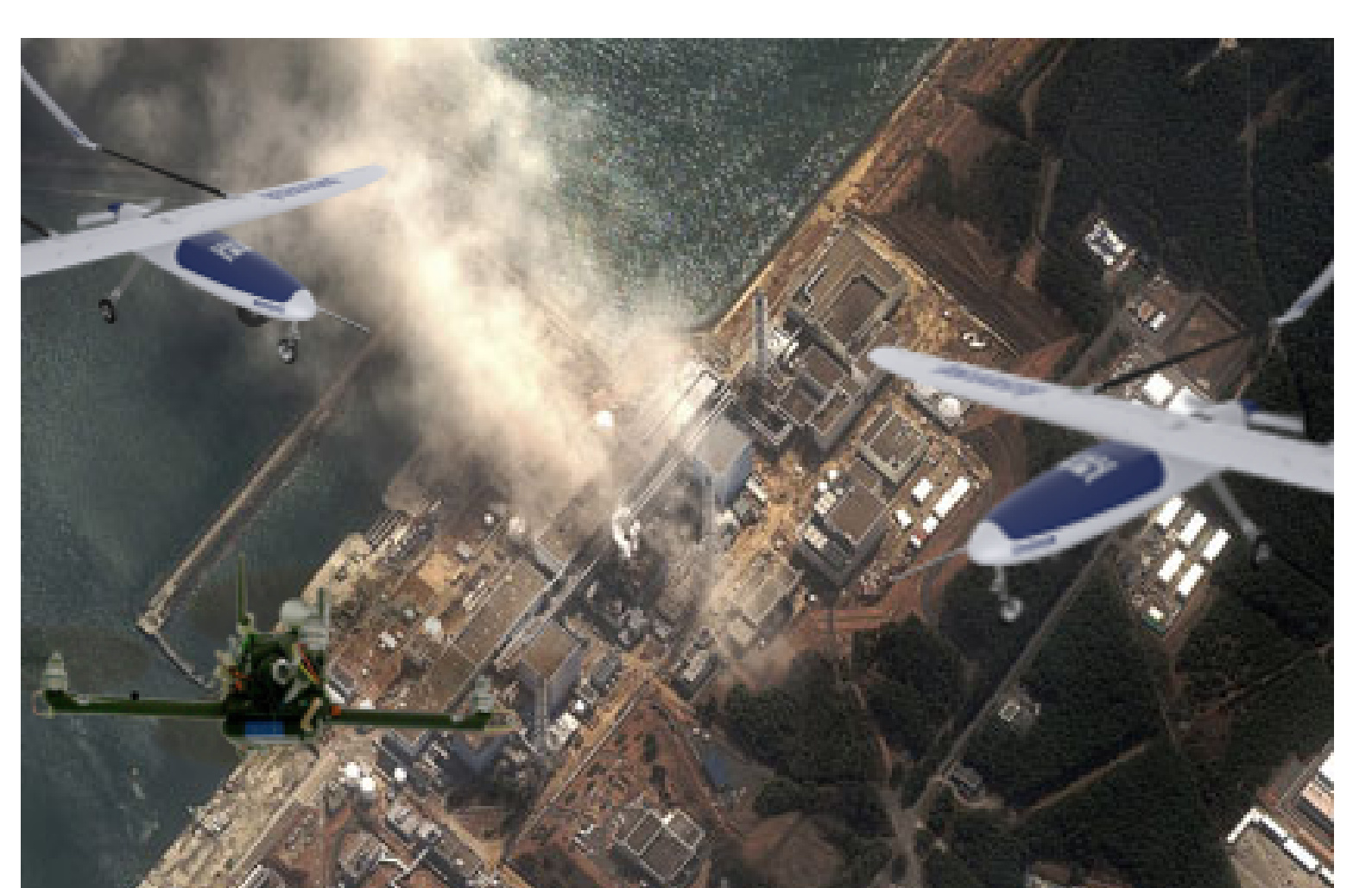

Estimate

$\rightarrow$\begin{tabular}{c|} 
State/Parameter \\
Estimation
\end{tabular}

Estimate Statistics
While most of the proposed AO strategies are centralized, distributed approaches (all calculations on-board) allow for more flexibility and avoid a central point of failure. We develop a new distributed approach, incorporating a process PDE-model instead of a simple analytical or qualitative model. The use of model order reduction is highly essential in this context as on-board computational power is limited and results must be obtained in real-time.

\section{Problem Description}

- Advection-diffusion equation to model dispersion of pollutant with concentration $c(\mathbf{x}, t)$ :

$$
\frac{\partial c}{\partial t}+\nabla \cdot(c \mathbf{u})-\nabla \cdot(\mathbf{D} \nabla c)=s, \quad c(\mathbf{x}, 0)=c_{0}(\mathbf{x})
$$

- Discretized form (FEM with characteristic Galerkin approach):

$$
\frac{\mathbf{Q}_{1} \mathbf{c}^{k+1}-\mathbf{Q}_{2} \mathbf{c}^{k}}{\Delta t}+\mathbf{A} \mathbf{c}^{k+1}=\mathbf{s}^{k+1}, \quad \mathbf{c}^{0}=\mathbf{c}_{\mathbf{0}}
$$

- Known: velocity field $\mathbf{u}(\mathbf{x})$ and diffusion matrix $\mathbf{D}(\mathbf{x})$

- Unknown: initial condition $c(\mathbf{x}, 0)$ and source $s(\mathbf{x}, t)$

- Goal: Estimate current process state $\mathbf{c}^{k}$ and source term $\mathbf{s}^{k}$ repeatedly.

\section{Reduced Order Model}

- Assumption: Source can be represented by a linear combination of $M$ predefined radial basis functions $q_{i}(\mathbf{x})$ :

$$
\begin{aligned}
s(\mathbf{x}, t) \approx s_{q}(\mathbf{x}, t) & =\sum_{i=1}^{M} \theta_{i}(t) q_{i}(\mathbf{x}) \\
q_{i}(\mathbf{x}, t) & =e^{-a\left\|\mathbf{x}-\mathbf{x}_{i}\right\|^{2}}
\end{aligned}
$$

- The initial condition $c(\mathbf{x}, 0)$ can be considered as a result of an instantaneous source at $t=0$.

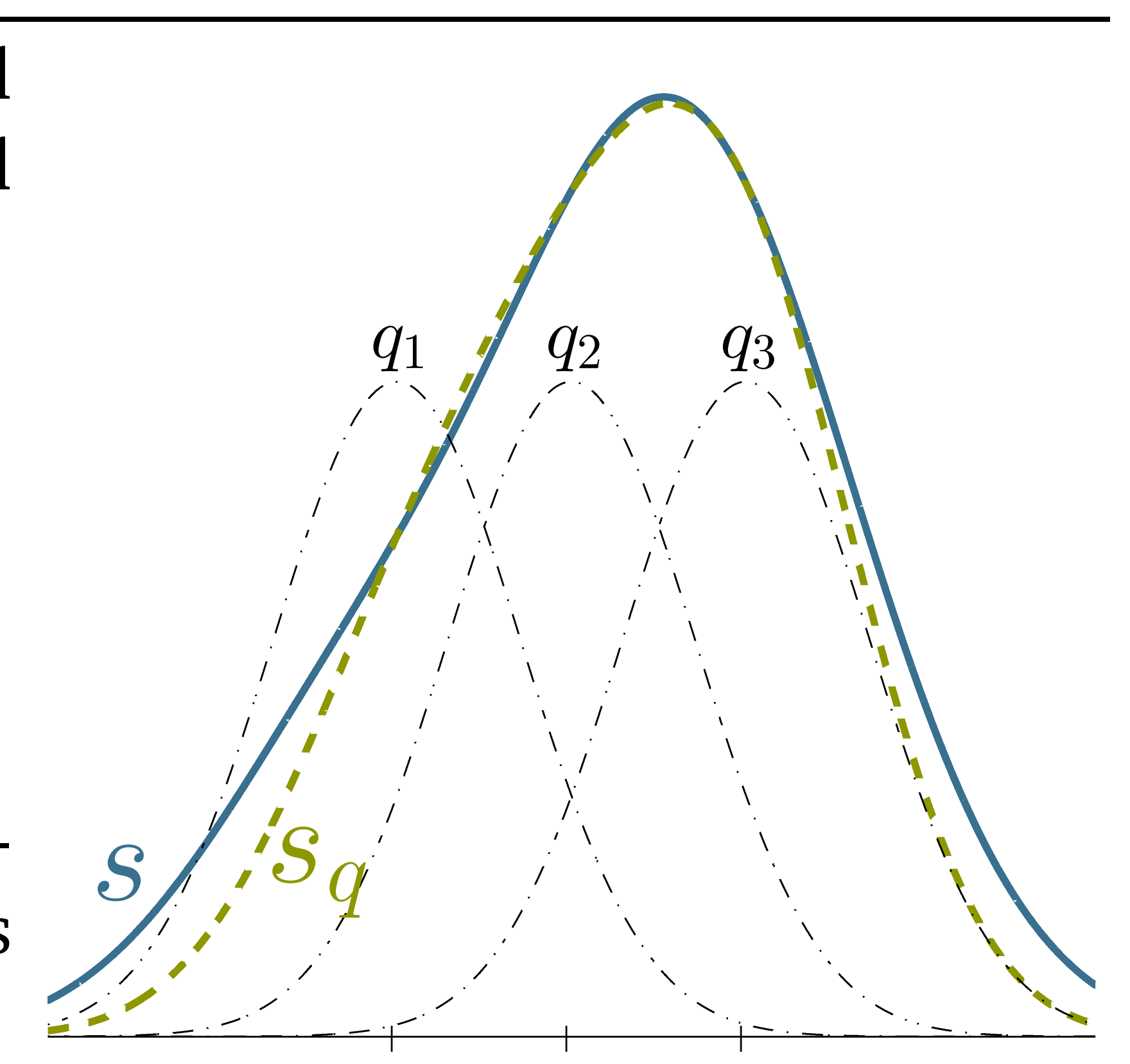

- If the system's impulse response $w_{i}(\mathbf{x}, t)$ corresponding to an excitation $s(\mathbf{x}, t)=q_{i}(\mathbf{x}) \delta_{0}(t)$ is known, the solution $c_{q}(\mathbf{x}, t)$ can be computed as

$$
c_{q}(\mathbf{x}, t)=\sum_{i=1}^{M} \int_{0}^{t} \theta_{i}(\tau) w_{i}(\mathbf{x}, t-\tau) d \tau \text {. }
$$

- For this reason, a snapshot set is built up by solving for every $\mathbf{q}_{i}$

$$
\frac{\mathbf{Q}_{1} \mathbf{w}_{i}^{k+1}-\mathbf{Q}_{2} \mathbf{w}_{i}^{k}}{\Delta t}+\mathbf{A w}_{i}^{k+1}=0 \quad \mathbf{w}^{1}=\frac{1}{\Delta t}\left(\frac{1}{\Delta t} \mathbf{Q}_{\mathbf{1}}+\mathbf{A}\right)^{-1} \mathbf{q}_{i} .
$$

- Proper Orthogonal Decomposition together with Galerkin projection yields the Reduced Order Model

$$
\frac{\tilde{\mathbf{Q}}_{\mathbf{1}} \tilde{\mathbf{c}}^{k+1}-\tilde{\mathbf{Q}}_{\mathbf{2}} \tilde{\mathbf{c}}^{k}}{\Delta t}+\tilde{\mathbf{A}} \tilde{\mathbf{c}}^{k+1}=\tilde{\mathbf{s}}^{k+1}, \quad \tilde{\mathbf{c}}^{0}=\tilde{\mathbf{c}}_{\mathbf{0}}
$$

with $\operatorname{dim}(\tilde{\mathbf{c}}) \ll \operatorname{dim}(\mathbf{c})$.

\section{ntegration of Measurements}

- Estimate current reduced process state $\tilde{\mathbf{c}}^{k,+}$ and parameters $\boldsymbol{\theta}^{k,+}$ from measurements $\mathbf{z}^{k}$ and model predictions $\tilde{\mathbf{c}}^{k,-}$ and $\boldsymbol{\theta}^{k,-}$.

- Dual Kalman Filter:

$$
\begin{aligned}
\tilde{\mathbf{c}}^{k,+} & =\tilde{\mathbf{c}}^{k,-}+\tilde{\mathbf{K}}_{c}^{k}\left(\mathbf{z}^{k}-\tilde{H}^{k}\left[\tilde{\mathbf{c}}^{k,-}\right]\right) \\
\boldsymbol{\theta}^{k,+} & =\boldsymbol{\theta}^{k,-}+\tilde{\mathbf{K}}_{\theta}^{k}\left(\mathbf{z}^{k}-\tilde{H}^{k}\left[\tilde{\mathbf{c}}^{k,-}\right]\right)
\end{aligned}
$$

- $\tilde{\mathbf{K}}_{c}^{k}$ depends on error statistics and measurement location, $\tilde{\mathbf{K}}_{\theta}^{k}$ additionally depends on sensitivity of the system regarding $\theta_{i}^{k}$.

- Maintenance of error covariance matrix describing uncertainties

- Complexity of filter algorithm $\sim \mathscr{O}\left(n^{2}\right) \Rightarrow$ large benefit with reduced models

\section{Results}

- 2D test case with Poiseuille flow and sinusoidal source function

- 15 radial basis functions, 9 sensors, 2262 DOF (original), 100 DOF (reduced) $\Rightarrow$ online speed-up (for filter) $>100$
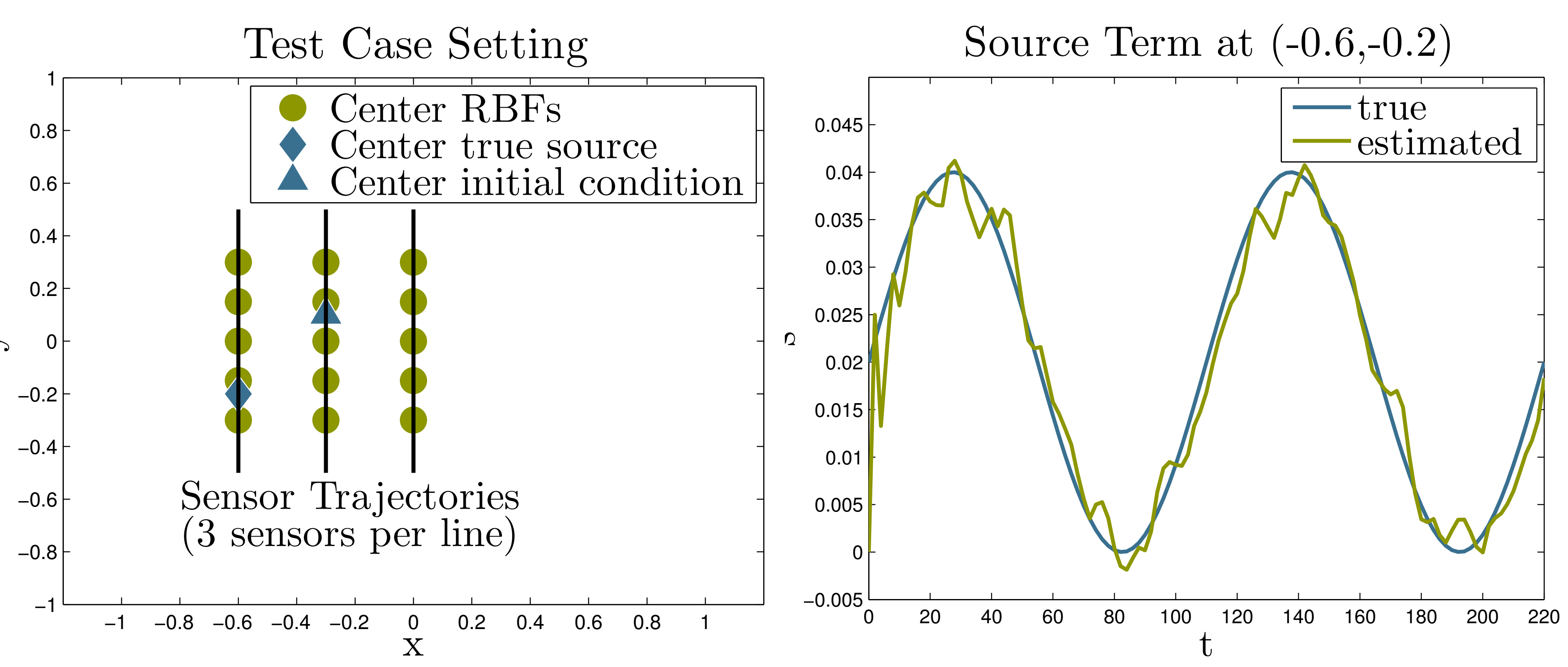

Est. Concentration $(t=220)$
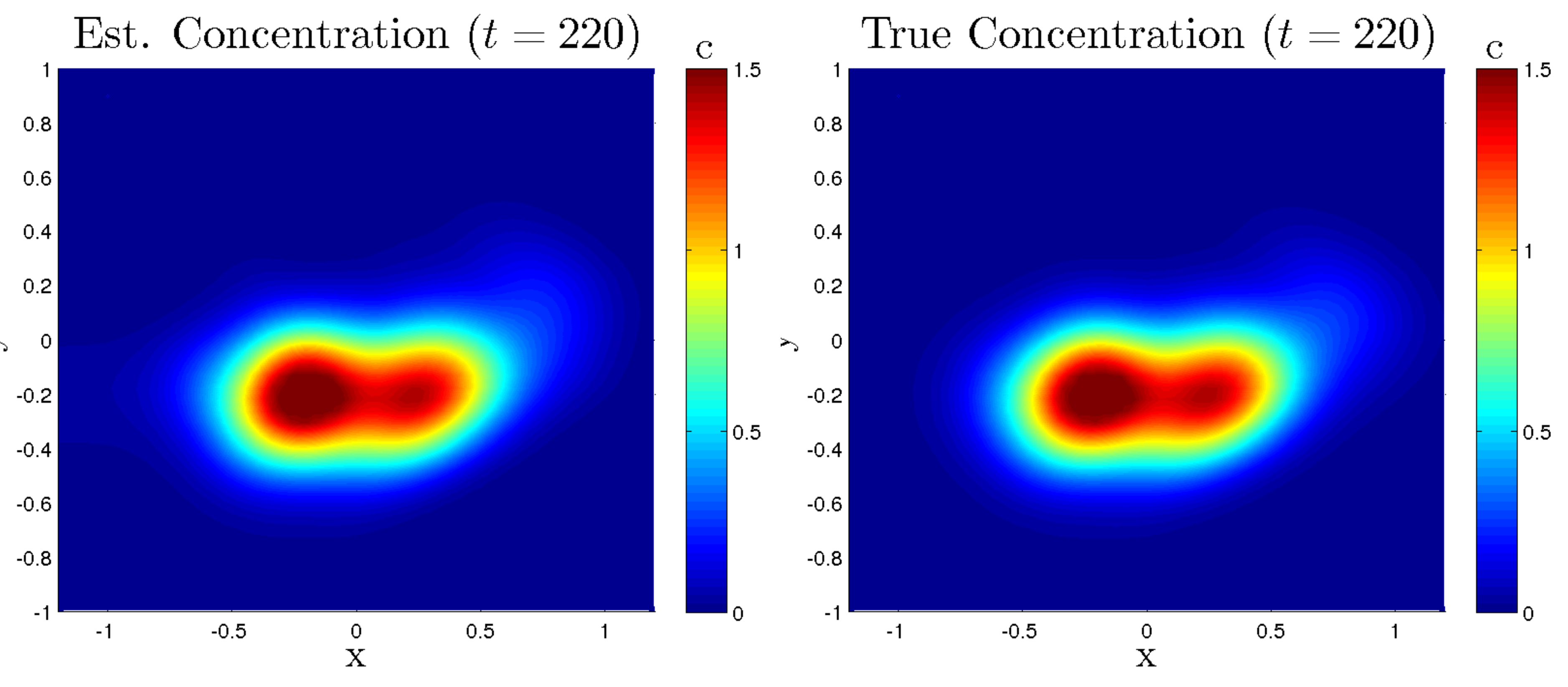

\section{Conclusion and Outlook}

- Reduced order models help to meet real-time requirements in monitoring of atmospheric dispersion processes.

- Outlook: AO strategy to improve estimates and reduce number of sensors

- based on error covariance matrices, cooperation, MPC ... 